\title{
Experimental Study on Axial Dependence of Anode Current Distribution in an Oven Magnetron
}

\section{AUTHOR(S):}

Mitani, Tomohiko; Shinohara, Naoki; Matsumoto, Hiroshi; Aiga, Masayuki; Kuwahara, Nagisa; Ishii, Takeshi

\section{CITATION:}

Mitani, Tomohiko ... [et al]. Experimental Study on Axial Dependence of Anode Current Distribution in an Oven Magnetron. IEEE Transactions on Electron Devices 2010, 57(5): 1167-1172

\section{ISSUE DATE:}

2010-05

URL:

http://hdl.handle.net/2433/120952

\section{RIGHT:}

(c) 2010 IEEE. Personal use of this material is permitted. Permission from IEEE must be obtained for all other users, including reprinting/ republishing this material for advertising or promotional purposes, creating new collective works for resale or redistribution to servers or lists, or reuse of any copyrighted components of this work in other works. 


\title{
Experimental Study on Axial Dependence of Anode Current Distribution in an Oven Magnetron
}

\author{
Tomohiko Mitani, Member, IEEE, Naoki Shinohara, Member, IEEE, Hiroshi Matsumoto, Fellow, IEEE, \\ Masayuki Aiga, Nagisa Kuwahara, and Takeshi Ishii
}

\begin{abstract}
The axial dependence of the anode current distribution in a $2.45-\mathrm{GHz}$ oven magnetron was investigated. We previously demonstrated that installing a cathode shield is an effective way to reduce magnetron noise. The objective of the present study is to determine why installing a cathode shield reduces the noise. We measured the anode current of a test magnetron with an axially segmented anode block. When no magnetic field was applied, the test magnetron behaved like a cylindrical vacuum diode, and the axial electron distribution corresponded to the axial distribution of the cathode temperature. When a magnetic field was applied, the cathode shield increased the normalized anode current in the central region, which contributes to noise reduction.
\end{abstract}

Index Terms-Cathodes, magnetrons, noise.

\section{INTRODUCTION}

A MAGNETRON is a well-known crossed-field vacuum device commonly used as a microwave oven heating source. A 2.45-GHz oven magnetron has a high dc-RF conversion efficiency of over $70 \%$, but it also has wideband oscillation, harmonics, $\pi-1$-mode oscillation, and spurious noise over a wide frequency range. Additional filtering circuits and noise shielding are thus necessary for microwave ovens to prevent electromagnetic interference with other electrical appliances. Several methods for reducing magnetron noise have been proposed and experimentally demonstrated.

Installing an ion-drain electrode in an oven magnetron effectively suppresses noise generated by ions trapped in the vicinity of the cathode [1]. Magnetic priming [2]-[5] and electric priming [6] reduce start-up noise by hastening $\pi$-mode selection. Installing a metal cathode shield has been also investigated as an effective method for reducing magnetron noise. Kohsaka et al. have proposed installing cathode shields on both ends of the cathode [7] to reduce magnetron noise. We have experimentally verified that installing a cathode shield on only the high-voltage (HV) input side of the cathode is more effective than installing

Manuscript received September 29, 2009; revised January 28, 2010. First published March 15, 2010; current version published April 21, 2010. This paper was supported in part by the Ministry of Education, Culture, Sports, Science and Technology (MEXT), Japan, through a Grant-in-Aid for Scientific Research, Young Scientists (B) under Grant 19760565. The review of this paper was arranged by Editor W. Menninger.

T. Mitani, N. Shinohara, and H. Matsumoto are with the Research Institute for Sustainable Humanosphere, Kyoto University, Kyoto 611-0011, Japan (e-mail: mitani@rish.kyoto-u.ac.jp).

M. Aiga, N. Kuwahara, and T. Ishii are with Panasonic Semiconductor Discrete Devices Company, Ltd., Tochigi 321-3231, Japan.

Digital Object Identifier 10.1109/TED.2010.2043385

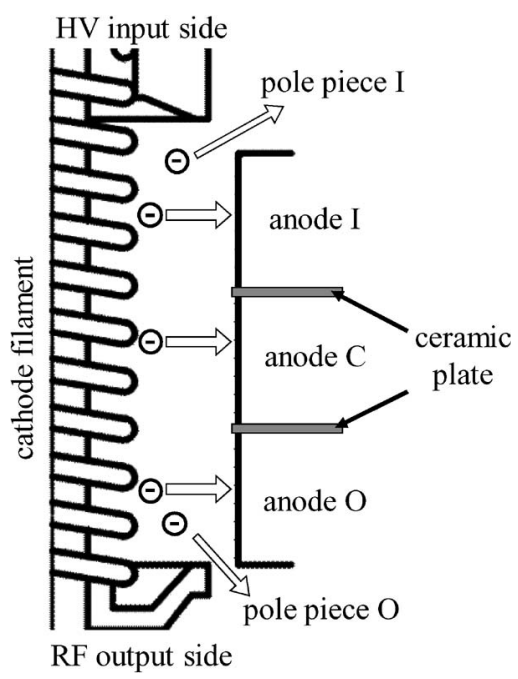

Fig. 1. Cross-sectional schematic view of the test magnetron with axially segmented anodes. The anode block of the test magnetron consists of five pieces: pole piece $\mathrm{I}$, anode $\mathrm{I}$, anode $\mathrm{C}$, anode $\mathrm{O}$, and pole piece $\mathrm{O}$.

two cathode shields on both ends of the cathode [8]. Although it causes about $3 \%$ degradation of the magnetron efficiency compared to a conventional oven magnetron, the cathode shield is easy to assemble into the magnetron filament and significantly suppresses the sideband noise around the carrier frequency, the $\pi-1$-mode oscillation, and spurious noise over a wide frequency range, which can be seen in [8].

The objective of the present study is to determine how a cathode shield reduces the noise. We measured the anode current of a test magnetron with axially segmented anode blocks, in order to estimate the axial dependence of the anode current in an oven magnetron.

\section{Measurement Configurations}

\section{A. Test Magnetron}

Figs. 1 and 2 show a cross-sectional schematic view and a photograph of the test magnetron, respectively. The labels "HV input side" and "RF output side" in Figs. 1 and 2 indicate the side on which the cathode filament is connected to the input power source and the side on which the RF output probe is located, respectively. The anode block of the test magnetron consists of five segments that surround the cathode filament: the pole piece on the HV input side (pole piece I), the anode block on the HV input side (anode I), the anode block in the 


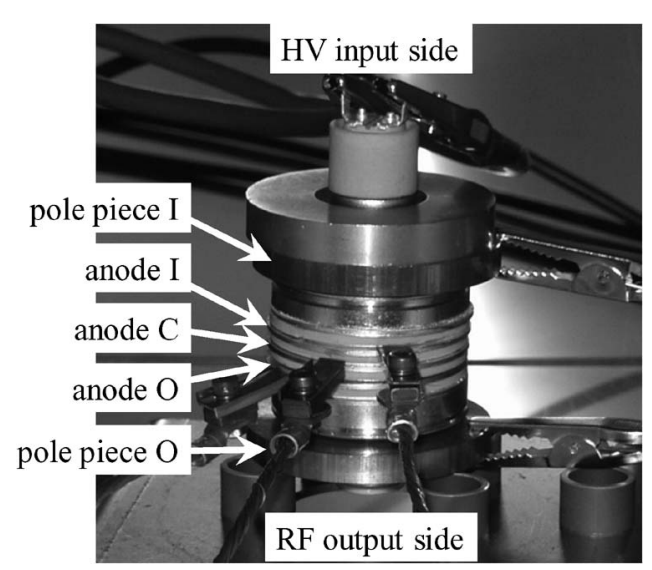

Fig. 2. Photograph of the test magnetron.

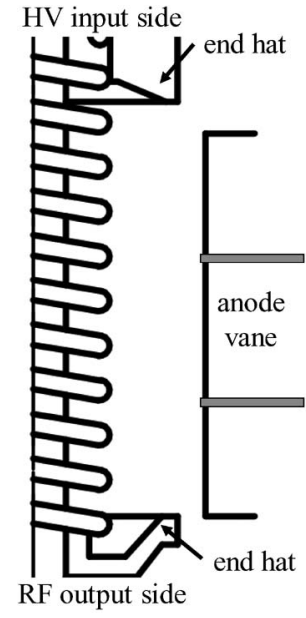

(a)

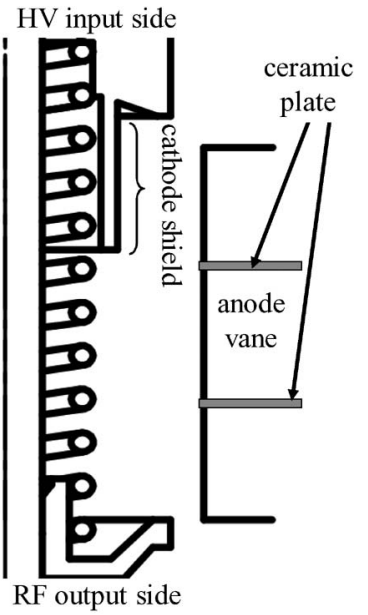

(b)
Fig. 3. Schematic cross-sectional views of the test magnetron cathodes. (a) Conventional cathode. (b) Shielded cathode.

central region (anode $\mathrm{C}$ ), the anode block on the RF output side (anode $\mathrm{O}$ ), and the pole piece on the RF output side (pole piece $\mathrm{O}$ ). Anode $\mathrm{I}$, anode $\mathrm{C}$, and anode $\mathrm{O}$ all have an axial length of $3 \mathrm{~mm}$. Each anode segment or pole piece is insulated from adjacent anode segment(s) by a ceramic plate to permit the anode current collected by each anode segment to be independently measured.

Two types of magnetron cathodes were examined: a conventional cathode and a shielded cathode. Cross-sectional schematic views of the cathodes are shown in Fig. 3. The configuration of the shielded cathode is the same as that depicted in [8].

Two cases were examined with respect to the external magnetic field: no applied magnetic field and an applied magnetic field of $0.11 \mathrm{~T}$. When no magnetic field is applied, the test magnetron is expected to function like a cylindrical vacuum diode. The magnetic field of $0.11 \mathrm{~T}$ is generated by permanent magnets attached to both pole pieces. The magnetic field strength of $0.11 \mathrm{~T}$, which is much smaller than that of commercially available oven magnetrons (approximately $0.18 \mathrm{~T}$ ), was selected in order to prevent the test magnetron from oscillating. Hence, oscillation of the test magnetron did not occur during the measurements.

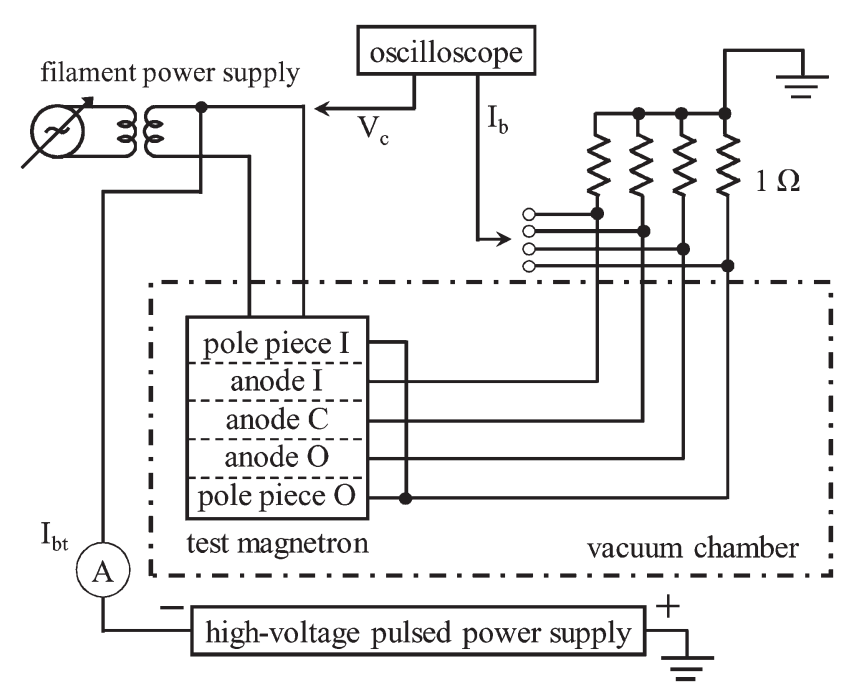

Fig. 4. Schematic of the measurement system. $V_{c}$ is the operating voltage, $I_{b}$ is the anode current, and $I_{\mathrm{bt}}$ is the total anode current.

\section{B. Measurement System}

Fig. 4 shows a schematic of the measurement system used for monitoring the anode current to each anode segment or pole piece. The test magnetron is completely enclosed in a vacuum chamber to evacuate the tube. An HV pulsed power supply operated the test magnetron at a pulse frequency of $50 \mathrm{~Hz}$ and a duty of 50\% with a constant filament current of $10 \mathrm{~A}$. Each anode segment or pole piece was connected to a resistance of $1 \Omega$, which was placed outside the vacuum chamber. The anode current $I_{b}$ collected on each anode segment or pole piece was obtained by measuring the voltage across each resistance using an oscilloscope. The total anode current $I_{\mathrm{bt}}$ was measured with an ampere meter. The operating voltage $V_{c}$ was also monitored with an oscilloscope.

\section{Measurement Results}

\section{A. Case I: No Magnetic Field}

With no magnetic field applied, Fig. 5(a) and (b) shows the measurement results for the anode current distribution as a function of the operating voltage $V_{c}$ for the conventional cathode and the shielded cathode, respectively. The total anode current $I_{\mathrm{bt}}$ is $3.2 \mathrm{~A}$ in the conventional cathode case and $5.1 \mathrm{~A}$ in the shielded cathode case, both at the operating voltage of $-5.0 \mathrm{kV}$.

As seen in Fig. 5(a) and (b), the anode current saturates at the operating voltage from -2.0 to $-5.0 \mathrm{kV}$ in the conventional cathode case and from -3.9 to $-5.0 \mathrm{kV}$ in the shielded cathode case. These results indicate that the test magnetron was operated in the temperature-limited regime, where the current is determined by the cathode filament temperature and the work function of the filament material, independent of the operating voltage. Fig. 5(a) and (b) also shows that the anode current is dependent on the operating voltage when the operating voltage is $-0.9 \mathrm{kV}$ in the conventional cathode case and from -0.9 to $-2.9 \mathrm{kV}$ in the shielded cathode case. We confirmed that the test magnetron enters the space-charge-limited regime, 


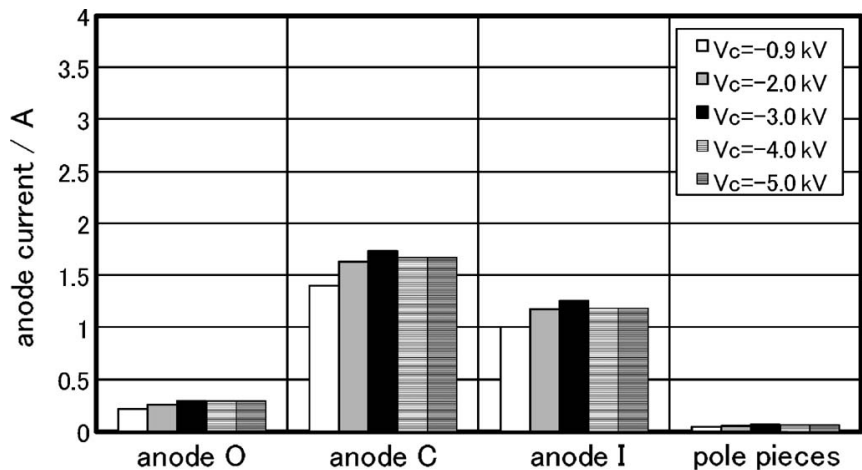

(a)

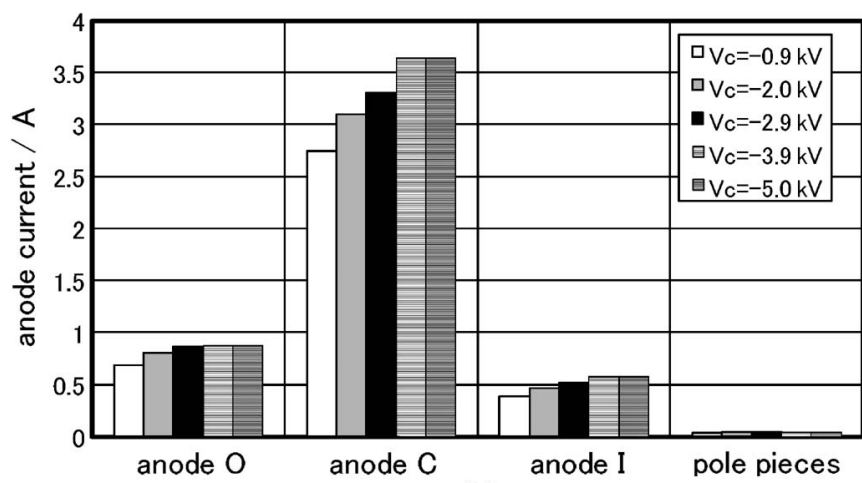

(b)

Fig. 5. Measured anode current distribution with no magnetic field applied. $V_{c}$ is the operating voltage. (a) Conventional cathode (b) Shielded cathode.

according to the Child-Langmuir law [9], in these operating voltage ranges. Hence, we verified that the test magnetron functions like a cylindrical vacuum diode when no magnetic field is applied, as predicted in Section II-A.

Fig. 6(a) and (b) shows the anode current distribution of the test magnetron normalized by the total anode current $I_{\mathrm{bt}}$ for the conventional cathode and the shielded cathode, respectively. Installing the cathode shield causes the anode current to the central region to increase from $50 \%$ to $70 \%$ of the total anode current, whereas the anode current to the HV input side is reduced from $40 \%$ to $10 \%$ of the total anode current.

\section{B. Case II: Applied Magnetic Field of 0.11 T}

Figs. 7 and 8 show the measurement results for the anode current distribution and the anode current distribution normalized by the total anode current $I_{\mathrm{bt}}$, respectively, as a function of the operating voltage. For both Figs. 7 and 8, the labels (a) and (b) indicate the results for the conventional cathode and the shielded cathode, respectively. The total anode current $I_{\mathrm{bt}}$ is 5.1 A in the conventional cathode case and 1.8 A in the shielded cathode case, respectively, both at the operating voltage of $-5.0 \mathrm{kV}$. The relationship observed between the operating voltage and the total anode current indicates that the test magnetron was operated in the space-charge-limited regime when a magnetic field was applied.

As when no magnetic field was applied, a comparison of Fig. 8(a) and (b) shows that the anode current collected by anode $\mathrm{C}$ (central anode) is increased by shielding the cathode,

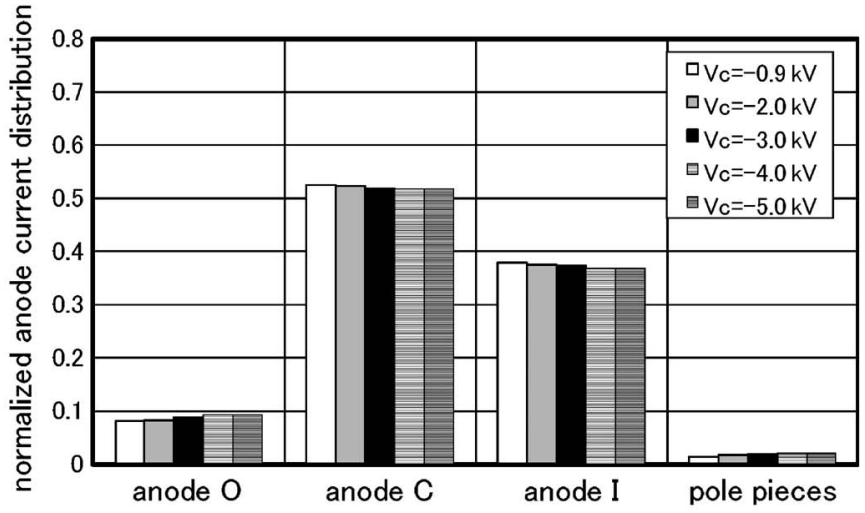

(a)

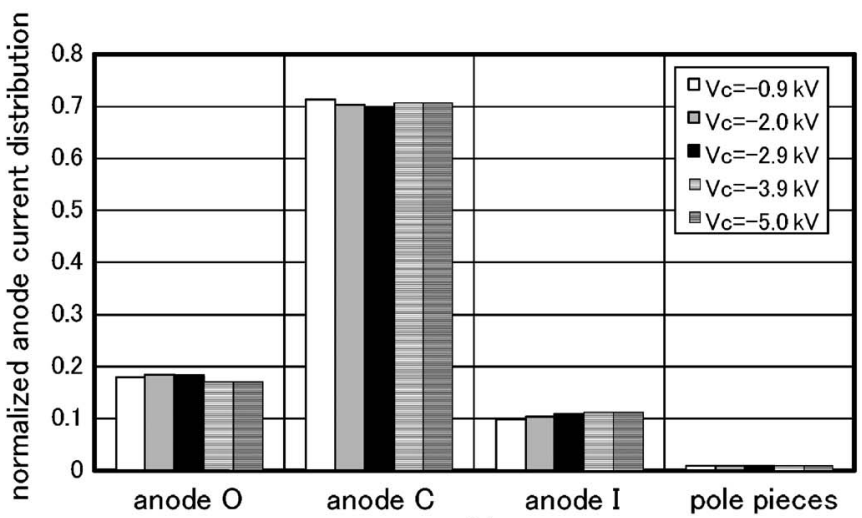

(b)

Fig. 6. Normalized anode current distribution (relative to the total anode current) with no magnetic field applied. $V_{c}$ is the operating voltage. (a) Conventional cathode. (b) Shielded cathode.

whereas the anode current collected by anode I (HV input side) is reduced.

\section{DISCUSSION}

\section{A. Validity of the Test Magnetron Measurements}

The measurement results reveal that when no magnetic field is applied to the test magnetron, it functions like a cylindrical vacuum diode in the temperature-limited regime. In this case, the current density of the electron emission $J$ is derived from the Richardson-Dushman equation, i.e.,

$$
J=A T_{K}^{2} \exp \left(-e \phi / k T_{K}\right)
$$

where $A$ is the Richardson constant, $T_{K}$ is the cathode temperature, $e$ is the electron charge, $k$ is the Boltzmann constant, and $\phi$ is the work function of the cathode material. Carburized thoriated tungsten was used for the cathode filament of the test magnetron. We previously measured the axial distribution of $T_{K}$ (see [8]). The average filament temperatures in the regions adjacent to anode $\mathrm{O}$, anode $\mathrm{C}$, and anode I were measured to be approximately 1710,1890 , and $1870 \mathrm{~K}$, respectively. The normalized current densities emitted from the three regions are calculated to be $10 \%, 49 \%$, and $41 \%$, respectively, which agrees reasonably well with the normalized anode current distribution shown in Fig. 6(a). This confirms that the test magnetron functions like a cylindrical vacuum diode when no magnetic field is applied. 


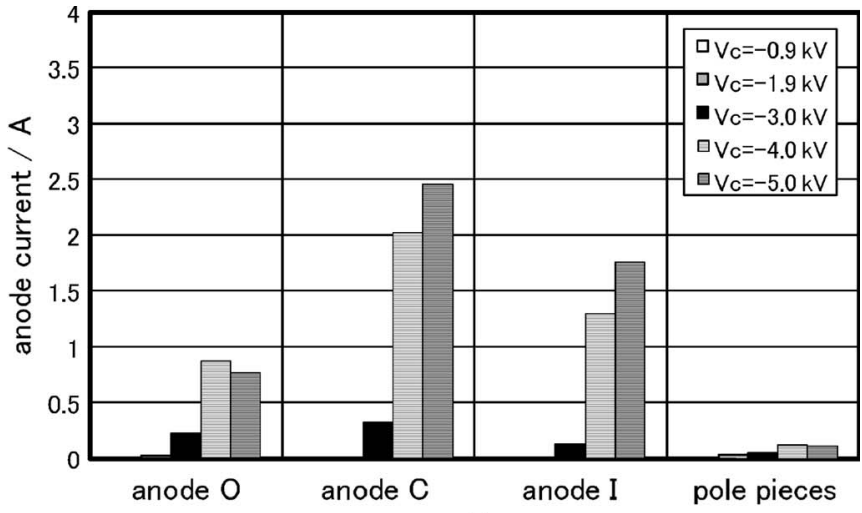

(a)

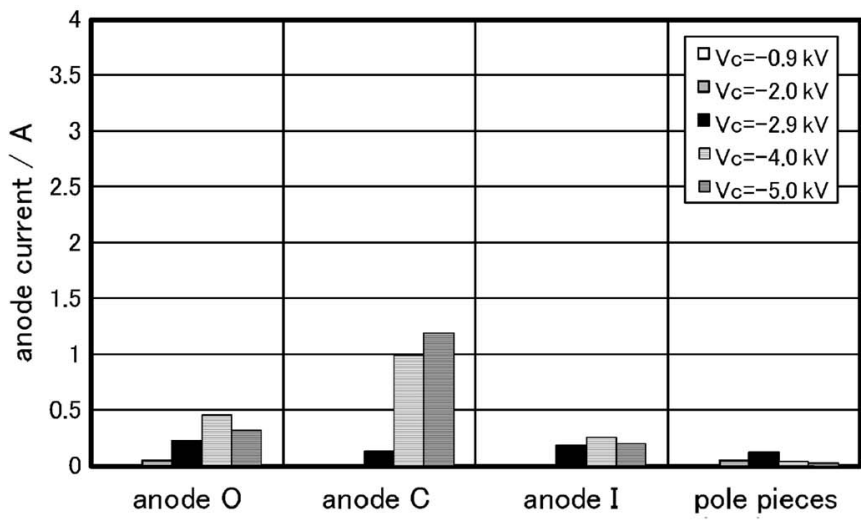

(b)

Fig. 7. Measured anode current distribution when a magnetic field of $0.11 \mathrm{~T}$ is applied. $V_{c}$ is the operating voltage. (a) Conventional cathode. (b) Shielded cathode.

The total anode current $I_{b t}$ differs between the conventional cathode case and the shielded cathode case, as mentioned in Section III. This is probably due to a difference in the filament temperatures. For example, the current density $J$ calculated using (1) decreases by $40 \%$ when the filament temperature is reduced from 1900 to $1850 \mathrm{~K}$. Although we have not yet measured the axial temperature distribution of the shielded cathode filament, such a small filament temperature difference lies within the specification range, and the measured total anode currents in both cases were large enough for magnetron operation.

\section{B. Leakage Current at Low Operating Voltages}

Of particular interest in Fig. 8 is the anode current distribution at low operating voltages of $-0.9,-1.9$, and $-2.9 \mathrm{kV}$. Note that a large amount of the anode current at the low operating voltages flows to the anode block on the RF output side (anode $\mathrm{O}$ ) and the pole pieces. Hence, this anode current can be regarded as leakage current, which is unnecessary for proper magnetron operation. In particular, the normalized leakage current to the pole pieces is quite high at an operating voltage of $-2.0 \mathrm{kV}$ for both the conventional and shielded cathodes. On the other hand, the leakage current to the pole pieces is less than $2 \%$ of the total anode current when no magnetic field is applied. Thus, the measurement results indicate that electrons

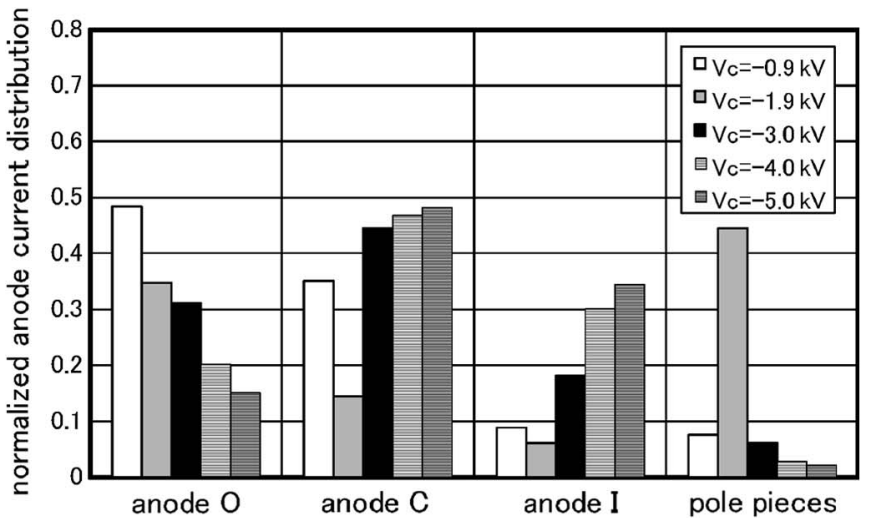

(a)

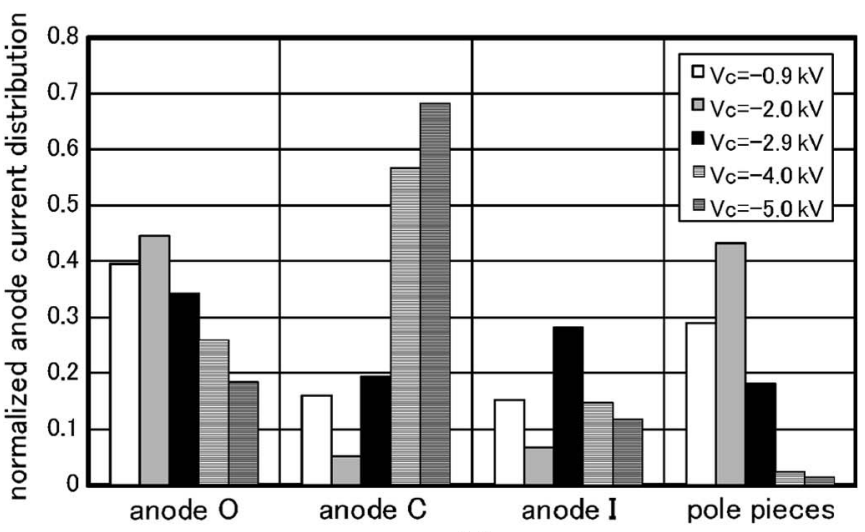

(b)

Fig. 8. Normalized anode current distribution (relative to the total anode current) when a magnetic field of $0.11 \mathrm{~T}$ is applied. $V_{c}$ is the operating voltage. (a) Conventional cathode. (b) Shielded cathode.

are diffused in the axial direction when a magnetic field is applied.

The leakage current to the pole pieces is attributed to the electric field generated by the space charge. The electron motion in a magnetron is governed by the Lorentz force and Poisson's equation. We assume that the RF electromagnetic field can be neglected at low operating voltages where oscillation does not occur, because electrons in the interaction space are affected little by the RF field. Under this assumption, the Lorentz force generated by the applied operating voltage, the applied magnetic field, and the electron density $\rho$ in the interaction space is given by

$$
m \frac{d \boldsymbol{v}}{d t}=-e(\boldsymbol{E}+\boldsymbol{v} \times \boldsymbol{B})
$$

and Poisson's equation can be written as

$$
\nabla \cdot \boldsymbol{E}=\rho / \varepsilon_{0}
$$

where $m$ is the electron mass, $\boldsymbol{E}$ is the electric field, $\boldsymbol{v}$ is the electron velocity, $\boldsymbol{B}$ is the applied magnetic field, and $\varepsilon_{0}$ is the electric permittivity. Note that the axial electron motion is ideally independent of $\boldsymbol{B}$ when a magnetic field is perfectly applied in the axial direction, i.e., $\boldsymbol{B}=B_{z}$, where $z$ indicates the axial direction.

Here, we analyze the axial diffusion effect derived from (2) and (3) in a magnetron on a planar electron beam, as depicted 


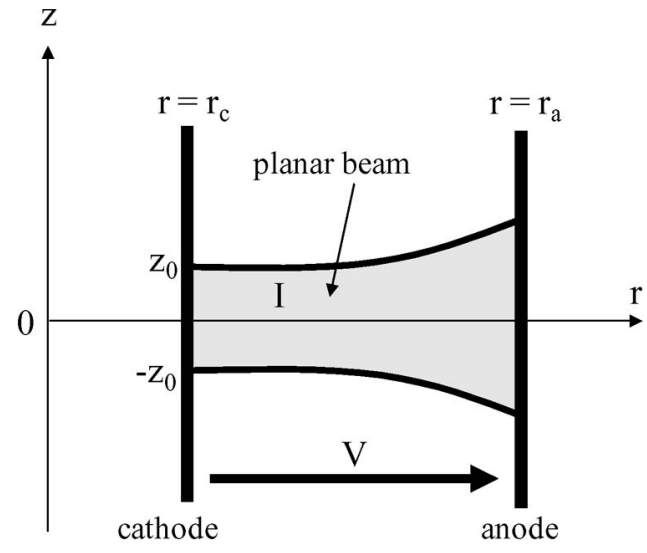

Fig. 9. Cross-sectional diagram of a planar beam. $r_{a}$ is the anode position, $r_{c}$ is the cathode position, $V$ is the anode-cathode voltage, $I$ is the current density, and $\pm z_{0}$ is the end position of the planar beam in the $z$-direction on the cathode.

in Fig. 9. Although this analysis only provides an order-ofmagnitude estimate, it allows us to analytically analyze the axial diffusion effect. The envelope curve of the planar beam in the $r z$ plane is expressed by the following equation [10], [11]:

$$
z= \pm \frac{I}{V^{\frac{3}{2}}} \frac{\sqrt{m / e}}{2^{\frac{7}{2}} \varepsilon_{0}}\left(r-r_{c}\right)^{2} \pm z_{0}
$$

where $r_{a}$ is the anode position, $r_{c}$ is the cathode position, $V$ is the anode-cathode voltage, $I$ is the current density in the $r$-direction, and $\pm z_{0}$ is the end position of the planar beam in the $z$-direction on the cathode, as shown in Fig. 9. Utilizing the measurement data, $V$ is the operating voltage at the anode radius $r_{a}$, and $I$ is roughly estimated to be $300 \mathrm{~A} / \mathrm{m}$ by dividing the total anode current by the cathode length (about $10 \mathrm{~mm}$ ) when no magnetic field is applied. From the test magnetron case, substituting $I=300 \mathrm{~A} / \mathrm{m}, V=1 \mathrm{kV}$, and $\left(r_{a}-r_{c}\right)=$ $2.5 \mathrm{~mm}$ into (4) results in an electron axial diffusion length on the order of $1 \mathrm{~mm}$ for the case when no magnetic field is applied. When a magnetic field is applied, electrons are diffused much further in the axial direction, because the effective travel time from the cathode to the anode is much longer than when no magnetic field is applied (i.e., the cylindrical diode case). Thus, when a magnetic field is applied, a significant amount of leakage current is expected to reach the pole pieces, as the measurement confirms.

The relationship between the observed leakage current at low operating voltages and start-up noise generation is still under investigation; however, the method presented in this paper for measuring the leakage current using segmented anode blocks should prove valuable in understanding how electron motion in the axial direction affects start-up noise in relativistic magnetrons and oven magnetrons.

\section{Role of the Cathode Shield}

As shown in Figs. 6 and 8, the anode current is mostly concentrated in the central region of the anode when a metal cathode shield is installed. In other words, the cathode shield prevents electrons from flowing to the ends, opposing the effect of axial diffusion.
Here, we discuss the electron motion in the vicinity of the anode ends. The electric field due to the operating voltage consists of radial and axial components at both anode ends due to end effects at the anode block or the cathode end hats, whereas it is aligned in the radial direction in the central region of the anode. Magnetron oscillation occurs through synchronization between the RF field generated by the anode resonant cavities and the $\boldsymbol{E} \times \boldsymbol{B}$ drift motion of electron spokes. Hence, pure oscillation must arise in the region where the electric and magnetic field are aligned in the radial and axial directions, respectively. In contrast, undesired electric or magnetic fields will create electron motions that will generate undesirable RF noise.

\section{CONClusion}

The anode current distribution has been measured in an oven magnetron to evaluate the role of the cathode shield. A test magnetron with and without a cathode shield has been measured. In each case, the magnetron under test has been measured both with no applied magnetic field and with an applied magnetic field of $0.11 \mathrm{~T}$. Measurements show that the cathode shield increases the normalized anode current collected in the central anode region, where the electric and magnetic fields are almost ideally "crossed," and prevents electrons from flowing to the anode ends. This results in enhanced pure oscillation and reduced unwanted noise. Measurements also show that an applied magnetic field can result in a significantly higher leakage current on the magnetron pole pieces due to greater axial diffusion of electrons. Future development may include confirmation of the noise reduction and minimization of startup noise using detailed particle-in-cell simulations.

\section{ACKNOWLEDGMENT}

Experimental measurements were conducted in the anechoic radio wave chamber of the Microwave Energy Transmission Laboratory (METLAB), which is one of the facilities of the cooperative study programs of the Research Institute of Sustainable Humanosphere, Kyoto University, Kyoto, Japan.

\section{REFERENCES}

[1] K. Yamamoto, H. Kuronuma, T. Koinuma, and N. Tashiro, "A study of magnetron noise," IEEE Trans. Electron Devices, vol. ED-34, no. 5, pp. 1223-1226, May 1987.

[2] V. B. Neculaes, R. M. Gilgenbach, and Y. Y. Lau, "Low-noise microwave magnetrons by azimuthally varying axial magnetic field," Appl. Phys. Lett., vol. 83, no. 10, pp. 1938-1940, Sep. 2003. DOI: 10.1063/1.1609040.

[3] V. B. Neculaes, R. M. Gilgenbach, Y. Y. Lau, M. C. Jones, and W. M. White, "Low-noise microwave oven magnetrons with fast startoscillation by azimuthally varying axial magnetic fields," IEEE Trans. Plasma Sci., vol. 32, no. 3, pp. 1152-1159, Jun. 2004.

[4] V. B. Neculaes, M. C. Jones, R. M. Gilgenbach, Y. Y. Lau, J. W. Luginsland, B. W. Hoff, W. M. White, N. M. Jordan, P. Pengvanich, Y. Hidaka, and H. L. Bosman, "Magnetic perturbation effects on noise and startup in DC-operating oven magnetrons," IEEE Trans. Electron Devices, vol. 52, no. 5, pp. 864-871, May 2005.

[5] V. B. Neculaes, M. C. Jones, R. M. Gilgenbach, Y. Y. Lau, J. W. Luginsland, B. W. Hoff, W. M. White, N. M. Jordan, P. Pengvanich, Y. Hidaka, and H. L. Bosman, "Magnetic priming effects on noise, startup, and mode competition in magnetrons," IEEE Trans. Plasma Sci., vol. 33, no. 1, pp. 94-102, Feb. 2005. 
[6] J. I. Kim, J. H. Won, G. S. Park, H. J. Ha, and J. C. Shon, "Reduction of noise in strapped magnetron by electric priming using anode shape modification," Appl. Phys. Lett., vol. 88, no. 22, p. 221 501, May 2006. DOI: $10.1063 / 1.2208273$

[7] A. Kohsaka, H. Saitoh, and T. Kawaguchi, "Low profile and clean spectrum magnetron," J. Microw. Power Electromagn. Energy, vol. 24, no. 1, pp. 3-13, 1989.

[8] T. Mitani, N. Shinohara, H. Matsumoto, M. Aiga, N. Kuwahara, and T. Ishii, "Noise reduction effects of oven magnetron with cathode shield on high voltage input side," IEEE Trans. Electron Devices, vol. 53, no. 8, pp. 1929-1936, Aug. 2006.

[9] I. Langmuir, "The effect of space charge and residual gases on thermionic currents in high vacuum," Phys. Rev., vol. 2, no. 6, pp. 450-486, Dec. 1913.

[10] P. T. Kirstein, G. S. Kino, and W. E. Waters, Space-Charge Flow. New York: McGraw-Hill, 1967, ch. 3.

[11] J. Ishikawa, Science and Technology of Charged Particle Beams, (in Japanese). Tokyo, Japan: Corona Publishing Co., Ltd., 2001.

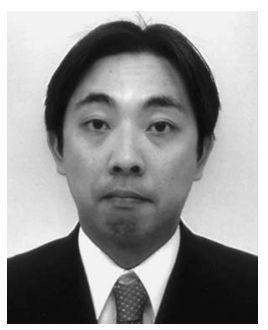

Tomohiko Mitani (M'05) received the B.E. degree in electrical and electronic engineering, the M.E. degree in informatics, and Ph.D. (Eng.) degree in electrical engineering from Kyoto University, Kyoto, Japan, in 1999, 2001, and 2006, respectively.

$\mathrm{He}$ is currently with Kyoto University, where he became an Assistant Professor with the Radio Science Center for Space and Atmosphere in 2003 and has been an Assistant Professor with the Research Institute for Sustainable Humanosphere since 2004. His current research interests include the experimental study on magnetrons, microwave heating applications, and microwave power transmission systems.

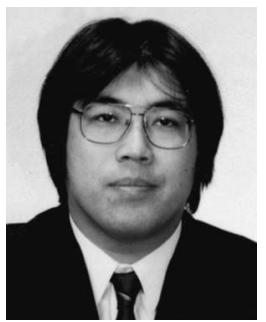

Naoki Shinohara (M'06) received the B.E. degree in electronic engineering and the M.E. and Ph.D. (Eng.) degrees in electrical engineering from Kyoto University, Kyoto, Japan, in 1991, 1993, and 1998, respectively.

He is currently with Kyoto University, where he became a Research Associate with the Radio Atmospheric Science Center in 1998 and with the Radio Science Center for Space and Atmosphere in 2000. In 2001, he became an Associate Professor. Since 2004, he has been an Associate Professor with the Research Institute for Sustainable Humanosphere, Kyoto University. He has been engaged in research on solar power station/satellite systems and microwave power transmission systems.

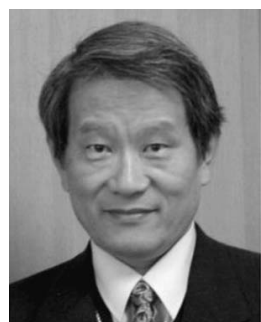

Hiroshi Matsumoto (F'03) received the B.E. degree in electronic engineering and the M.E. and Ph.D. (Eng.) degrees in electrical engineering from Kyoto University, Kyoto, Japan, in 1965, 1967, and 1973, respectively.

In 1977, he became a Professor with the Radio Atmospheric Science Center, Kyoto University, where he was the Director from 1992 to 1998. In 2000, he became a Professor with the Radio Science Center for Space and Atmosphere, Kyoto University, where he was the Director from 2002 to 2004. He is currently a Professor with the Research Institute for Sustainable Humanosphere, Kyoto University, where he was the Director from 2004 to 2005. He was a member of the Board of Directors, Kyoto University, from 2005 to 2008 and has been the President of Kyoto University since 2008. He has been engaged in research on space plasma physics, space radio science and engineering, solar power station/satellite systems, and microwave power transmission systems.

Dr. Matsumoto was named an RAS-Associate of the Royal Astronomical Society, U.K., in 2004. He is a member of the American Geophysical Union and the Society of Geomagnetism and Earth, Planetary and Space Sciences (SGEPSS). He was the President of the International Union of Radio Science (URSI) from 1999 to 2002.

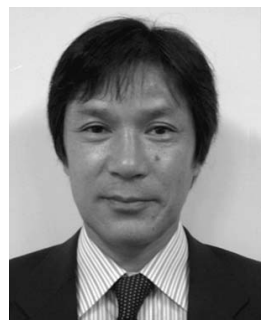

Masayuki Aiga received the degree from Owase Industrial High School, Owase, Japan, in 1970.

$\mathrm{He}$ was with the Matsushita Electronic Instruments Corporation from 1999 to 2005. He joined Panasonic Semiconductor Discrete Devices Company, Ltd., Tochigi, Japan, through the merger of the companies. He has been engaged in development of magnetrons.

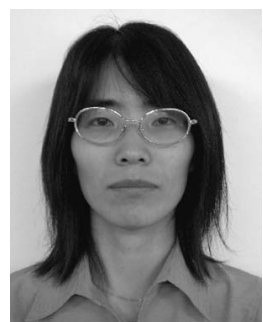

Nagisa Kuwahara received the B.E. degree in electronic engineering from Utsunomiya University, Utsunomiya, Japan, in 1989.

She was with the Matsushita Electronic Instruments Corporation from 1989 to 2005. She joined Panasonic Semiconductor Discrete Devices Company, Ltd., Tochigi, Japan, through the merger of the companies. She has been engaged in development of magnetrons.

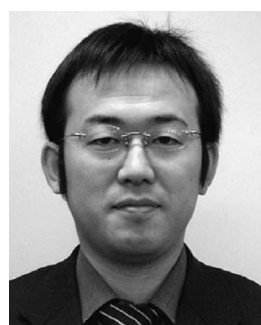

Takeshi Ishii received the B.E. degree in electronic engineering from Ashikaga Institute of Technology, Ashikaga, Japan, in 1998.

He was with the Matsushita Electronic Instruments Corporation from 1998 to 2005. He joined Panasonic Semiconductor Discrete Devices Company, Ltd., Tochigi, Japan, through the merger of the companies. He has been engaged in development of magnetrons. 